\title{
Molar Pregnancy along with Normal Fetus - A Case Report
}

\author{
NAHREEN AKHTAR ${ }^{1}$, SABINA KARIM ${ }^{2}$
}

\begin{abstract}
:
The extremely rare condition of molar pregnancy with a coexisting fetus progressing to a viable infant, is reported. At 32 wks, prenatal diagnosis was made by biochemical and sonographic findings in which elevated free ß-human chorionic gonadotrophic was noted and ultrasound showed a well defined multicystic snow storm like mass connecting with normal placenta. At 37 weeks cesarean section was done due to gestational hypertension, fetal growth restriction and $\mathrm{H} / \mathrm{O}$ previous one cesarean section. A $2.3 \mathrm{~kg}$ male living boy was delivered. The infant did not show any abnormality. The placenta and the connecting hydatidiform mole were delivered. A normal B HCG level was noted in subsequent follow up.
\end{abstract}

Key words: complete mole, live fetus, ß human chorionic gonadotropin

\section{Introduction:}

Gestational trophoblastic disease consists of a broad spectrum of conditions ranging from an uncomplicated partial hydatidiform molar pregnancy to stage IV choriocarcinoma with cerebral metastasis. The modified World Health Organisation classification of gestational trophoblastic disease includes Hydatidiform mole which includes complete mole, partial mole and invasive mole. Second group include trophoblastic tumor like lesion which include exaggerated placental site reaction and placental site nodules. Third group include trophoblastic tumour, which include choriocarcinoma, placental site trophoblastic tumour (PSTT), epitheliod trophoblastic tumor. The usual management of gestational trophoblastic disease is evacuation of the uterus and follow up because higher chances of patient to develop choriocarcinoma. ${ }^{1}$ But some time when molar change is there in the placenta along with an alive fetus then expectant management can be performed under strict surveillance. ${ }^{2,3}$ Incidence of a live fetus associated with a hydatidiform mole is extremely rare. Hydatidiform mole without a fetus in ultrasonogram has a reported incidence of 1 in 2000 pregnancies and the incidence of hydatidiform mole with a coexisting fetus varies from $1 ; 10,000$ to 1 in 100,000 pregnancies. ${ }^{4}$ We present a case who was diagnosed to have hydatidiform mole along with live fetus diagnosed at second trimester and was referred to us.

\section{Case Report:}

A 31- year- old woman, Para $1 \mathrm{C} / \mathrm{S}+0$, hailing from Bonosree Dhaka was referred from a private clinic at her 32wks of pregnancy. She had no complaint on arrival other than $\mathrm{H} / \mathrm{O}$ threatened abortion at early pregnancy. She did not give any history of excessive vomiting in her early pregnancy. She is married for 12yrs . She had regular menstrual cycles prior to her pregnancy. Her last menstrual period was on 17.9.14 and accordingly her EDD was on 24.6.15. She had an early USG for dating and also had two USG after her threatened abortion, all showed normal findings. She did not have any anomaly scan but had USG at her 32wks pregnancy which shows normal placenta along with hydatidiform mole. Her AFI was $8 \mathrm{~cm}$.Serum ß HCG was $1,21,985 \mathrm{mIU} / \mathrm{mL}$ (normal value -2700 78100). Her liver, renal and thyroid function were normal. Follow up serum BHCG was done which shows $86,970.5 \mathrm{mlU} / \mathrm{mL}(9.5 .15)$ and $62,155.80 \mathrm{mlU} /$ $\mathrm{ml}$ (27.5.15).Clinically she was mildly anemic and mildly hypertensive $(140 / 90 \mathrm{mmHg})$ and abdomen was enlarged and corresponds with her gestational age. She was then followed fortnightly and was uneventful. Cesarean section was done at her $37 \mathrm{wks}$ of pregnancy in a private clinic on the day of admission. A healthy male baby of $2.3 \mathrm{~kg}$ was born. There were two separate placenta one healthy and one placenta had complete molar change. She was discharged on $4^{\text {th }}$ postoperative day. One unit of compatible blood was

1. Professor Dr Nahreen Akhtar, Fetomaternal Medicine wing, Dept of Obs \& Gynae. BSMMU

2. Dr Sabina Karim, Resident phase B, Pediatric Oncology BSMMU 
transfused per operatively. Histopathology shows Hydatidiform mole. ß hCG was done weekly. Beta human chorionic gonadotropin (âhCG) was normal after two weeks of delivery. Follow up was done by serum ßhCG.The baby was also found healthy at 6weeks postnatal period.

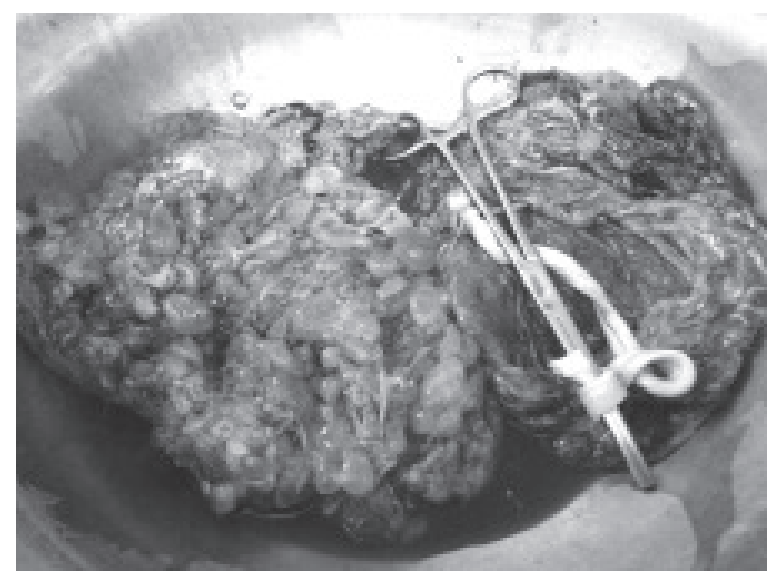

Photo showing on the right side there is normal placenta and on the left side molar change of placenta

\section{Discussion:}

Three different mechanism are possible, a complete mole (diploid,all paternal in origin) coexistent with a normal diploid fetus, a partial mole (triploid, maternal or paternal in origin) coexistent with a normal diploid fetus and a partial mole with an abnormal triploid fetus(both having 69 chromosomes). The first 2 mechanism represent dizogotic twin pregnancy, and the coexistent triploid fetus tends to die in first trimester. Hydatidiform mole with a coexistence fetus can be established by the partial mole syndrome or by a twin pregnancy where the other conceptus has degenerated into a mole. ${ }^{5}$ The current case was due to first mechanism. In our present case the histopatho-logical and morphological findings of a normal placenta and separated molar tissues with marked hydrophic avascular villi support the theoretical explanation that it resulted from a twin dizygotic pregnancy in which one twin had developed normally and the other had degenerated into a complete mole. Hydatidiform mole with alive fetus is a rare phenomenon. Few case were reported. Myamato reported a case, hydatidiform mole with live fetus. Diagnosis was done antenatally at $14 \mathrm{wks}$ of pregnancy, where pregnancy was terminated at $31 \mathrm{wks}$ gestation because of profuse pervaginal bleeding. A healthy baby of $1230 \mathrm{gm}$ were delivered. Baby shows no abnormality and had good development at the age of 6 months. Mass of molar tissue was completely separated from placenta. This suggest possibility of molar change in one of the twins. ${ }^{6}$ Unsal MA reported a case which was diagnosed at $14 \mathrm{wks}$ gestation but fetal death occurred at 20 wks gestation. The follow up period for two year was unremarkable. ${ }^{7}$ The typical sonographic findings of a molar pregnancy should be differentiated from a missed abortion with gestational sac especially during early pregnancy or even a partially necrotic fibroid can produce similar appearance. ${ }^{8}$ The difference between partial and complete mole cannot be firmly established by ultrasound because they both present vesicular pattern. In our case the ultrasound clearly revealed normal fetus and normal placenta coexisting with molar tissue. Since the normal placenta separate from molar tissues can be well defined, a complete mole with a concurrent fetus can be diagnosed prenatally. It is important to distinguish between a complete mole and partial mole when a fetus coexsist, because it has been reported that a complete mole has $20 \%$ chance to become invasive mole or even a choriocarcinoma, when the risk is far less in partial mole. ${ }^{9}$ Here HCG titre is generally higher than is seen in nonmolar gestation. Khazeli et al suggested that excessive production of free BHCG may indentify gestational trophoblastic disease. In present case HCG was also higher than corresponding gestational age. However relationship between HCG and coexisting fetus has not been reported. ${ }^{9}$ Therefore, the use of ultrasound, augmented by the significantly elevated HCG, and clinical symptoms and signs has facilitated the accurate prenatal diagnosis of coexsistent mole and fetus. Molar pregnancy with a coexsisting fetus progressing to a viable healthy infant is an extreme rarity. In most of these cases, the pregnancy is terminated due to severe complications such as vaginal bleeding, preeclampsia and missed abortion. ${ }^{10}$ Some recommended immediate termination even in the absence of any complication. As a result, management of molar pregnancy with coexsisting fetus remains controversial. In the present case diagnosis was done at 32 wks pregnancy so decision was taken for continuation of pregnancy after proper councelling. Although we demonstrate that a complete molar pregnancy with a normal concurrent fetus can permit the normal development of the fetus until term with a good fetal and maternal outcome, we cannot ignore the high risk of developing persistent gestational 
trophoblastic disease later, especially with the presence of a significantly high concentration of free ßHCG and complete mole. ${ }^{11}$ In present case there were single placenta probably fused one. One placenta was healthy and another one completely change into molar tissue. These findings strongly suggest the possibility of molar change in one of twins. Mothers serum HCG is within normal limit after six weeks of delivery. Still she will be in follow up upto five years.

Conclusion: Molar pregnancy coexistent with a live fetus is a rare event. Our case presented to us at late second trimester and was managed successfully with multidisciplinary approach. It was uneventful. Prenatal consultation should incude fetal and maternal risks, especially possible need for chemotherapy or even hysterectomy.It is also necessary to have close follow up and evaluation of patient during prenatal and postnatal period.

\section{References:}

1. Hancock BW,TidyJA. Current management of molar pregnancy. J Reprod Med 2002; 47(5): 347- 54.

2. Berkowitz RS, Tuncer ZS , Bernstein MR, Goldstein DP, Mangement of Gestational Trophoblastic disease. Subsequent Pregnancy experience. Semin oncol 2000; 27(6) : 678-85

3. Shapter AP, Mc Lellan R.Gestational trophoblastic disease. Obstet Gynecol Clin North Am 2001; 28(4): 805-17
4. SzulmanAE, Surti U.The syndromes Hydatidiform mole cytogenetics and morphologic correlations Am J Obstet Gynecol 1978 ; 132: $20-27$

5. Ozoktay S, Abel- Bey G, Alexander LL.Hydatidiform mole with a live fetus $\mathrm{J}$ of National Medical Association 1982 ;74(10): 1924-26

6. Miyamato $\mathrm{U}$, Katsuragama H, Ishiko J. J advances in Obstetrics \& Gynaecology 1989;41: (4) $561-3$

7. Unsal MA, Guven S. Complete Hydatidiform mole with a living fetus $J$ clinical and Experimental Obstetrics \& Gynaecology 2012 , $39(2): 262-4$

8. Sauerbree EE, Shia S, Fayle B.Coexsistent Hydatidiform mole and a normal fetus in the second trimester Radiology, 1980; 135: 415-17

9. Khazeli MB,Buchina ES, Pattillo RA. Radioimmunoassay of free $\beta$ subunit HCG in diagnosis of high and low risk gestational trophoblastic disease. Am J Obstet Gynecol 1989; 160:444- 449

10. JonesWB, Lauren NH. Hydatidiform mole with coexisting fetus. Am J Obstet Gynecol 1975;122: 267 -272.

11. ChenFP. Molar pregnancy and living normal fetus coexisting until term: prenatal biochemical and sonographic diagnosis -case report. Human Reproduction 1997 ; 12: 853- 856 